\title{
Comparison of the performance of analog and digital hadronic sampling calorimeters
}

\author{
Y. Elmahroug ${ }^{1 \star}$, B. Tellilit ${ }^{1,2}$ and S. Chedly ${ }^{3}$ \\ ${ }^{1}$ Unité de Recherche de Physique Nucléaire et des Hautes Energies, Faculté des Sciences de Tunis, \\ 2092 Tunis, Tunisie. \\ ${ }^{2}$ Université de Tunis El Manar, Institut Supérieur des Technologies Médicales de Tunis, 1006 Tunis, Tunisie. \\ ${ }^{3}$ Université de Carthage, École Polytechnique de Tunisie, B.P. 743 - 2078 La Marsa, Tunisie.
}

Accepted 29 January, 2013

\begin{abstract}
By using the tungsten as passive element and the Glass Resistive Plate Chambers (GRPCs) as active element, the gaseous hadronic calorimeter is studied as a possible detector for the future International Linear Collider (ILC). The results of the numerical study, using the GEANT4 simulation package, of the performance of this calorimeter when it is exposed to a negative pion beam in an energy range from 5 $\mathrm{GeV}$ to $100 \mathrm{GeV}$ are presented in this paper. Operating characteristics of this prototype, such as signal linearity, energy resolution and reconstructed energy have been simulated and examined. Moreover, a comparison between the Digital Hadron Calorimeter (DHCAL) and the Analog Hadron Calorimeter (AHCAL) is made.
\end{abstract}

Key words: Calorimetry, resistive plate chambers, international linear collider (ILC), GEANT4.

\section{INTRODUCTION}

Among the most important objectives of future particle physics experiments at the international linear collider (ILC) [ILC reference design report (RDR); ILC Homepage http://www.linearcollider.org/cms/.] are searching for one or more Higgs particles and the study of their characteristics. Many interesting physics processes will be provided by the electron-positron collisions at the center-of-mass energies between $500 \mathrm{GeV}$ and $1 \mathrm{TeV}$ in ILC such as $\mathrm{W}, \mathrm{Z}$ and $\mathrm{H}$ heavy bosons processes. These processes will be characterized by multi-jet. A very good energy resolution $(30 \% / \sqrt{E})$ is required to the reconstruction of heavy bosons $(\mathrm{W}, \mathrm{Z}, \mathrm{H})$ in hadronic final states (Doroba, 2004; Thomson, 2009).

The most efficient method to achieve this resolution is particle flow algorithm (PFA). This method allows to separate, respectively the deposited energies from photons and due to hadrons and the energies deposited from the charged hadrons and due to neutral hadrons in a jet. The optimal application of this technique requires

\footnotetext{
*Corresponding author. E-mail: youssef_phy@hotmail.fr.
}

highly granular hadronic calorimeter in order to separate the shower (Thomson, 2009). Accordingly, the study of a calorimeter performance is an essential step.

In this context, several kinds of Hadron Calorimeters (HCALs) are under construction and testing (Ammosov, 2002; Ammosov et al., 2004; Andreev et al., 2005, 2006). Among the best HCALs are those which use Resistive Plate Chambers (RPCs) as active elements. This is due to their several advantages compared to other detection technologies, such as their strength and their very good performances in terms of temporal and spatial resolutions (Repond, 2007; Drake et al., 2007; Bilki et al., 2008, 2009). Glass RPCs were chosen instead of bakelite RPCs due to their long-term stability, they do not need oil surface treatment with linseed oil, their fabricate is simple with low production cost and their homogeneity (Calcaterra et al., 2004; Candela et al., 2004; Akindinov et al., 2004; Ammosov et al., 2007; Drake et al., 2007; Va'vra, 2003; Ambrosio et al., 2003; Fonte, 2002).

In this paper, a study based on GEANT4 simulation is conducted to investigate the performance of a HCAL using tungsten as an absorber material and RPCs as active elements. The objective of this study is to simulate the energy resolution, the linearity and the reconstructed 


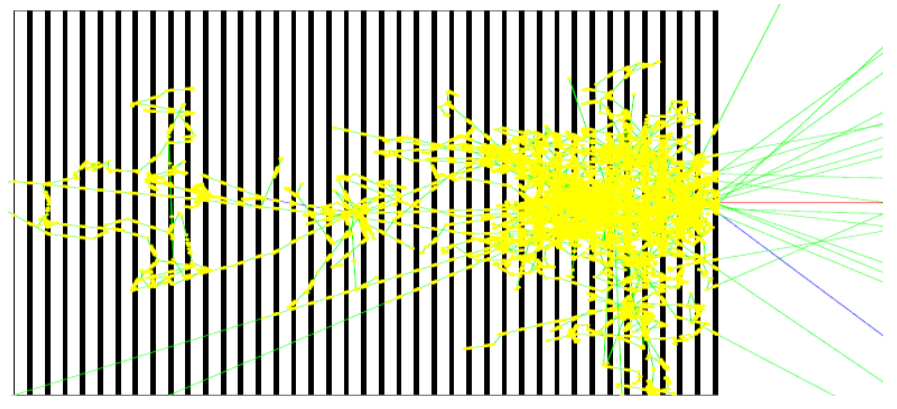

Figure 1. The calorimeter geometry for the simplified in GEANT4 with a $10 \mathrm{GeV}$ pion event. White layers represents the absorber, and the black layers are the sensitive material.

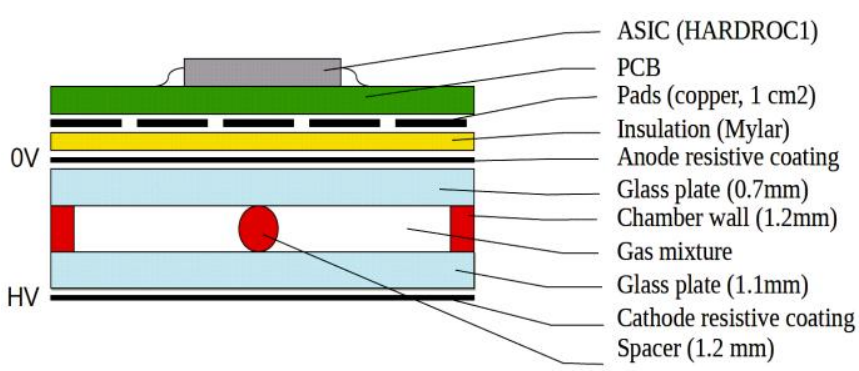

Figure 2. Schematic view of a glass RPC (Ammosov et al., 2007; Bedjidian et al., 2010).

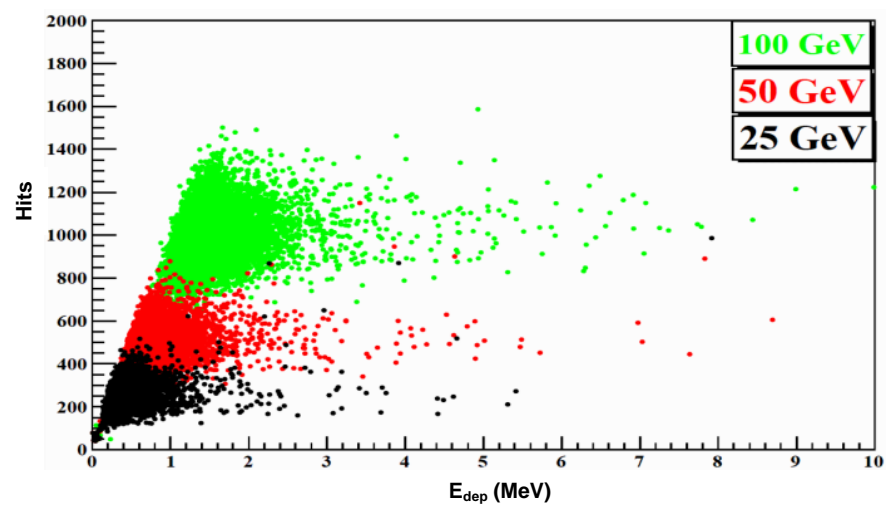

Figure 3. Correlation between the deposited energy and the hits number for 25, 50 and $100 \mathrm{GeV}$ pions energies.

energy of single pions and make a comparison between digital and analog methods.

\section{METHODOLOGY}

In this study, the HCAL has been simulated with a Monte Carlo program based on the GEANT4 (Geometry ANd Tracking) version 9.2.4 (Agostinelli et al., 2003; The GEANT4 web page: http://cern.ch/geant4).
This program is inspired from the examples of GEANT4 (novicelexempleNO3 and lovice lexempleN07, extended Ipersistency: P01 and extendedlelectromagneticlTestEm3 and advanced VAr calorimeter) and uses the ILC Physics. There is a list to simulate the interactions of particles with matter (ILC Physics List Homepage). For analysis of the simulated data by GEANT4, we developed programs based on software Root (ROOT, version 5.30.01. CERN, 2011).

The calorimeter used in this study consisted of 40 layers; each layer is composed of an absorber and a sensitive material. The dimensions of calorimeters are $100 \times 100 \times 104.16 \mathrm{~cm}^{3}$ and they are in such away that $95 \%$ of hadronic shower cascades energy is deposited in the calorimeter for $100 \mathrm{GeV}$ pion (Adloff et al., 2009; Leroy and Rancoita, 2000). The structure of calorimeter is shown in Figure 1. Each active layer is segmented into $1 \times 1 \mathrm{~cm}$ readout cells (pads) and a total of 400000 channels in 40 layers. The absorber is a layer of $2 \mathrm{~cm}$ of tungsten and the sensitive detector is a RPCs having a thickness of $6.04 \mathrm{~mm}$, these chambers consisted of two parallel glass plates spaced of $1.2 \mathrm{~mm}$, with a thickness of $0.7 \mathrm{~mm}$ on the anode side and $1.1 \mathrm{~mm}$ on the cathode one. Between these two plates, we insert a gas mixture composed of R134A (93\%), isobutane $(5.0 \%)$ and a sulfur-hexafluoride (2\%) (Camarri et al., 1998), and on the outer faces of the glass we put a graphite film with a thickness of $0.05 \mathrm{~mm}$ for the anode side and 0.06 for the cathodes one. Above graphite, we put a mylar layer with $0.05 \mathrm{~mm}$ thickness on the anode side and $0.18 \mathrm{~mm}$ on the cathode one. Finally, a printed circuit board (PCB) (G10) with $2.7 \mathrm{~mm}$ of thickness represents the readout electronics $(1.5 \mathrm{~mm})$ and the real PCB (1.2) is deposited on the anode of mylar. The structure of RPC is shown in Figure 2; for more details about the RPC structure see (Ammosov et al., 2007; Bedjidian et al., 2010).

We generated 10000 events of pion in the perpendicular direction of the calorimeter with energies from 5 to $100 \mathrm{GeV}$ with a step equal to $5 \mathrm{GeV}$. The first 5000 events are used to measure the deposited energy, estimate the number of hits and the rest is for assessing the reconstructed energy.

\section{ENERGY RESOLUTION AND LINEARITY}

\section{Deposited energy in analog and digital mode}

The first step for analysis of the data simulated by GEANT4 is to measure the total deposited energy by a primary pion in the active medium (the RPCS). This was done by summing the deposited energy in all cells hit (hits or pads) which have an energy greater than the 0.1 minimal ionizing particle (MIP). This concerns the analog mode. For the digital mode, instead of using the deposited energy, we counted the total number of hits which has a deposited energy higher than 0.1 MIP. In fact, the number of hits is proportional to the total deposited energy. This proportional relationship is shown in Figure 3.

The second step for data simulation is plotting and fitting the distributions of the measured energy and number of hits. From the fit parameters, we determined the linearity and the resolution. The distribution of the deposited energy and the distribution of the hits number are shown respectively in Figures 4 and 5 . We found that the deposited energy by primary pions follows a Landau distribution with right-hand tail due to the Landau fluctuation, but in the digital mode this fluctuation disappears, and the distribution follows a Gaussian distribution.

\section{Linearity}

As indicated previously, the fit parameters of deposited energy distributions and the hits number was used to estimate the linearity of the calorimeter response and the primary pions energy. Indeed, 


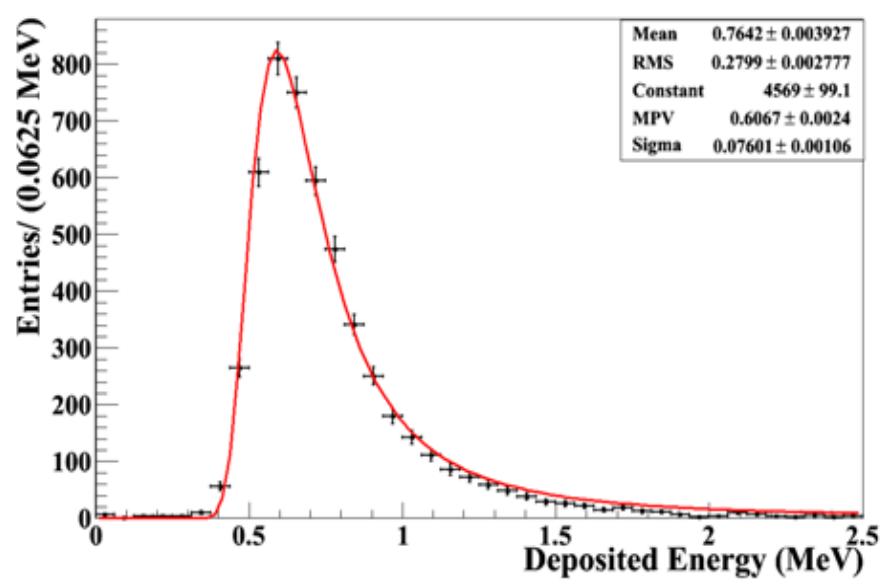

Figure 4. Distributions of deposited energy for $50 \mathrm{GeV}$ pions.

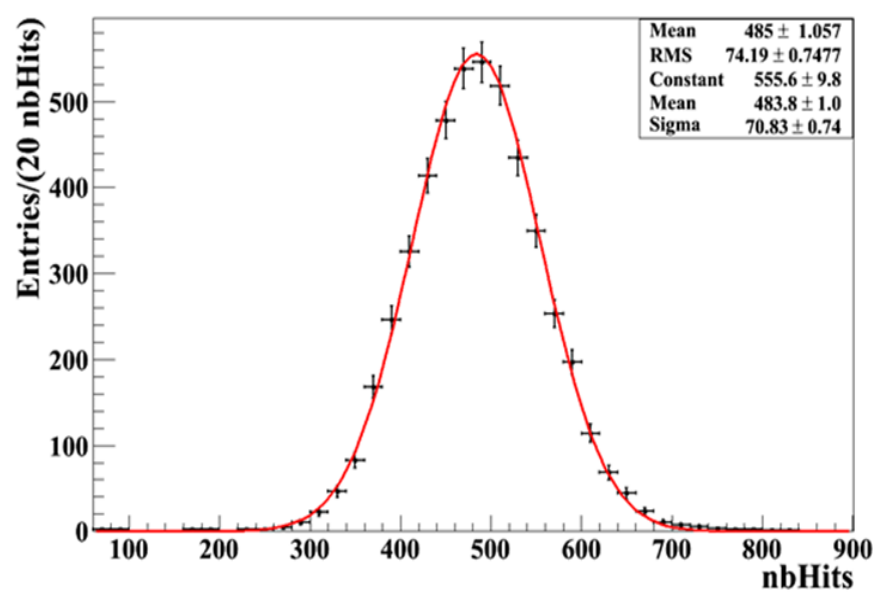

Figure 5. Distributions of hits number for $50 \mathrm{GeV}$ pions.

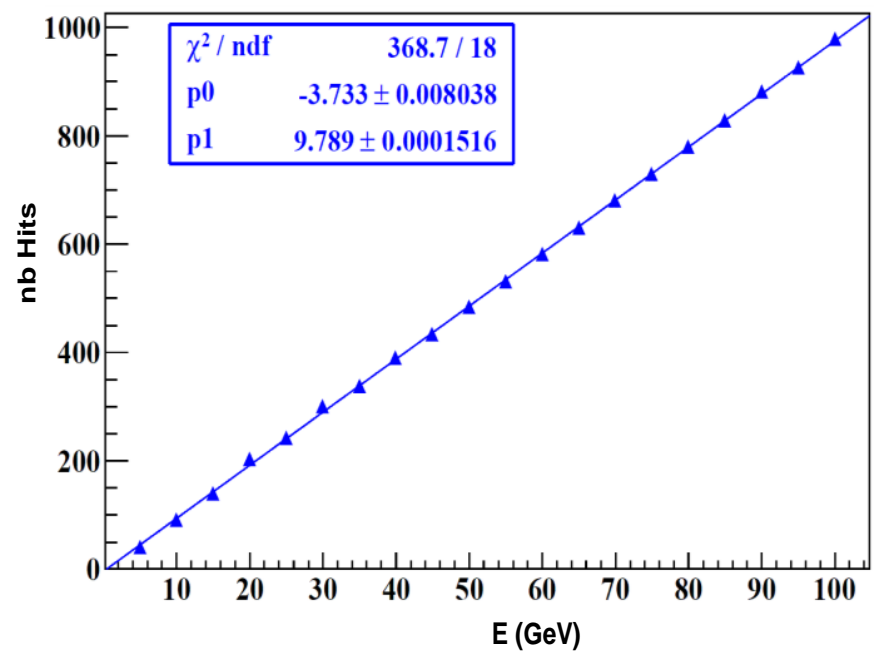

Figure 6. Linearity between the hits number and the primary pions energy for digital readout.

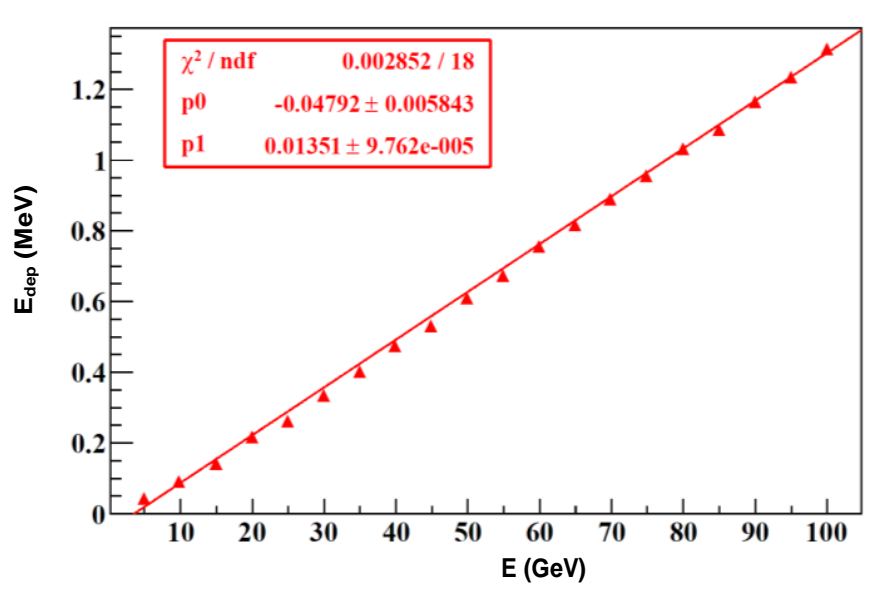

Figure 7. Linearity between the deposited energy and the primary pions energy for analog readout.

for each energy of primary pions, the average value of the Gaussian and Landau distributions was taken, then these values were plotted and fitted by a polynomial function of first degree $(p=$ $\left.p_{0}+p_{1} \cdot x\right)$. The linearity between the calorimeter response and the primary pions energy for digital and analog modes are shown, respectively in Figures 6 and 7. It was observed that the deposited energy and the hits number are proportional to the incident energy. By taking into account the fit parameters, the deposited energy and the hits number are as follows:

$E_{\text {dep }}(\mathrm{MeV})=(0.01351 \pm 0.00009762) E(\mathrm{GeV})-(0.04792 \pm 0.005843) E(\mathrm{GeV})$

$N_{\text {hit }}=(9.789 \pm 0.0001516) E(\mathrm{GeV})-(3.733 \pm 0.008038) E(\mathrm{GeV})$

\section{Resolution of the deposited energy and the hits number}

The same method used to estimate linearity was used to determine the energy resolution. The fit parameters of the deposited energy distribution or hits number were used. Since the resolution is defined as the ratio between the width at half height and the mean value, it was determined and then we plotted as a function of the primary pion energy. The fitting was done by the following resolution function:

$\frac{\sigma}{E}=\frac{S}{\sqrt{E}}+\frac{N}{E}+C$

With $\mathrm{S}$ the term of stochastic, $\mathrm{C}$ is a constant and $\mathrm{N}$ the electronic noise term is not taken into account because there is no electronic noise in Monte Carlo simulations. The energy resolution for digital and analog modes is presented in Figure 8. The fits values $\left(p_{0}=C\right.$ and $p_{1}=S$ ) are shown in Table 1 .

At low energies, the energy resolution in the digital mode is better than in the analog mode, this can be explained by the fact that in an analog calorimeter, the deposited energy by a pion in RPCs presents a large tail due to Landau fluctuations. These fluctuations increase the energy resolution for the analog readout compared to the digital case, because these effects are suppressed in the digital mode (Magill, 2003). However, for high energies, the degradation of the resolution in digital mode was observed which is caused by saturation effects of hits number. Indeed, at high incident energy, the shower particle density will be very high, and this increases the 


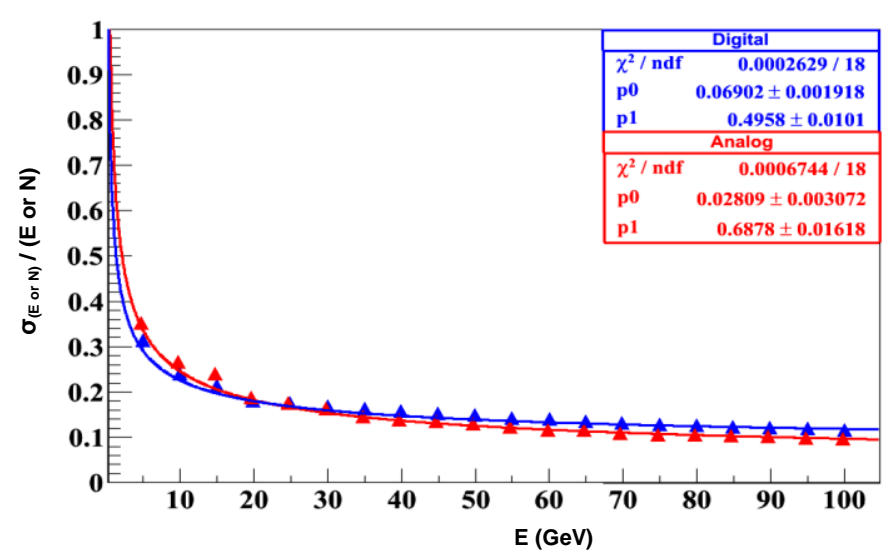

Figure 8. Comparison between the energy resolution of the digital readout and that of the analog $\left(p_{0}=C\right.$ and $\left.p_{1}=S\right)$.

Table 1. Parameters of energy resolution.

\begin{tabular}{lcc}
\hline Mode & Stochastic (\%) & Constant (\%) \\
\hline Digital & $49.58 \pm 1.01$ & $6.90 \pm 0.1918$ \\
Analog & $68.78 \pm 1.618$ & $2.81 \pm 0.3072$ \\
\hline
\end{tabular}

probability that a cell will be crossed by several secondary particles, but these particles will be counted as one hit (Wigmans, 2000).

\section{RECONSTRUCTED ENERGY}

\section{Distribution of reconstructed energy}

As previously discussed, the linear relationship between the calorimeter response and the incident pion energy can be translated by Equations 1 and 2. Using these two equations and replacing $E$ by $E_{r e c}$, the reconstructed energy can be written as follows:

$E_{\text {rec }}(G e V)= \begin{cases}(74.01924 \pm 0.53484) \times E_{\text {dep }}+(3.547002 \pm 0.458124) & \text { Analog } \\ \left(0.102155 \pm 1.58 \times 10^{-6}\right) \times N+\left(0.381346 \pm 8.2715 \times 10^{-4}\right) & \text { Digital }\end{cases}$

From these two equations, we determined the reconstructed energy of each primary pion. As noted previously, the reconstructed energy was calculated using events from 5000 to 10000 and to test and validate our simulation program. Figures 9 and 10 represent the reconstructed energy distributions using the deposited energy and the hits number for primary pions with energies of $50 \mathrm{GeV}$ for two different event groups. The first group is composed by the first 5000 events and the second is constituted by the events from 5000 to 10000 . It is clear that the distributions are similar for the two groups of events (parameters of the two distributions are very close). So, our program is valid and for the following work we will use only the second group of events.
It was also noted that the reconstructed energy distribution using the deposited energy has the same shape as that of the deposited energy distribution and also the reconstructed energy distribution using the hits number has the same shape as that of the hits number distribution.

\section{Response and linearity}

The linearity between reconstructed energy and primary pions energy is obtained by plotting the mean values of the reconstructed energy distribution according to the incident energy. These points are fitted by a polynomial of degree one. The linearity curves for digital and analog modes are shown respectively in Figures 11 and 12 . From the fit parameters, it was deduced that the reconstructed energy can be written in the following form:

$\mathrm{E}_{\mathrm{rec}}(\mathrm{GeV})=\left\{\begin{array}{l}(1.0009793 \pm 0.0004368) \mathrm{E}(\mathrm{GeV})+(0.0052804 \pm 0.0000992) \text { Analog } \\ (1.0036925 \pm 0.0011798) \mathrm{E}(\mathrm{GeV})+(0.0016034 \pm 0.0000711) \text { Digital }\end{array}\right.$

The calorimeter response can also be described by another variable called the nonlinearity which is defined by $\frac{\mathbf{E}_{\mathbf{T e c}}-\mathbf{E}}{\mathbf{E}}$, and presented in Figures 13 and 14 for two readouts. The maximum value of nonlinearity is about $1 \%$ for the analog mode and $3 \%$ for the digital mode. The linearity degradation in the digital mode is due to the saturation phenomenon. As earlier explained, at high incident energies, the number of counted hits is saturated; for this it does not follow the augmentation of incident energy (Wigmans, 2000).

\section{Resolution of reconstructed energy}

To determine the energy resolution $\frac{\boldsymbol{\sigma}_{\mathbf{E} \text { rec }}}{\mathbf{E}_{\mathbf{r e c}}}$, the same procedure which was used to calculate and plot theresolutions $\frac{\boldsymbol{\sigma}_{\mathbf{N}}}{\mathbf{N}}$ and $\frac{\boldsymbol{\sigma}_{\mathbf{E d e p}}}{\underline{E_{\text {dep }}}}$ was applied. The resolution for digital and analog modes is presented in Figure 15. As well as the deposited energy resolution $\frac{\mathbf{\sigma}_{\mathbf{E d e p}}}{\mathbf{E} \text { dep }}$ and the hits number $\frac{\boldsymbol{\sigma}_{\mathbf{N}}}{\mathbf{N}}$ at low energies, the digital mode provides a better resolution $\frac{\boldsymbol{\sigma}_{\mathbf{E r e c}}}{\mathbf{E}_{\mathbf{r e c}}}$. This is due to Landau fluctuations of the reconstructed energy in the analog case. At high energies, in the analog mode, the resolution is better than in the digital case because of the effects of hits number saturation (Wigmans, 2000; Magill, 2003). This resolution can be written as follows: 

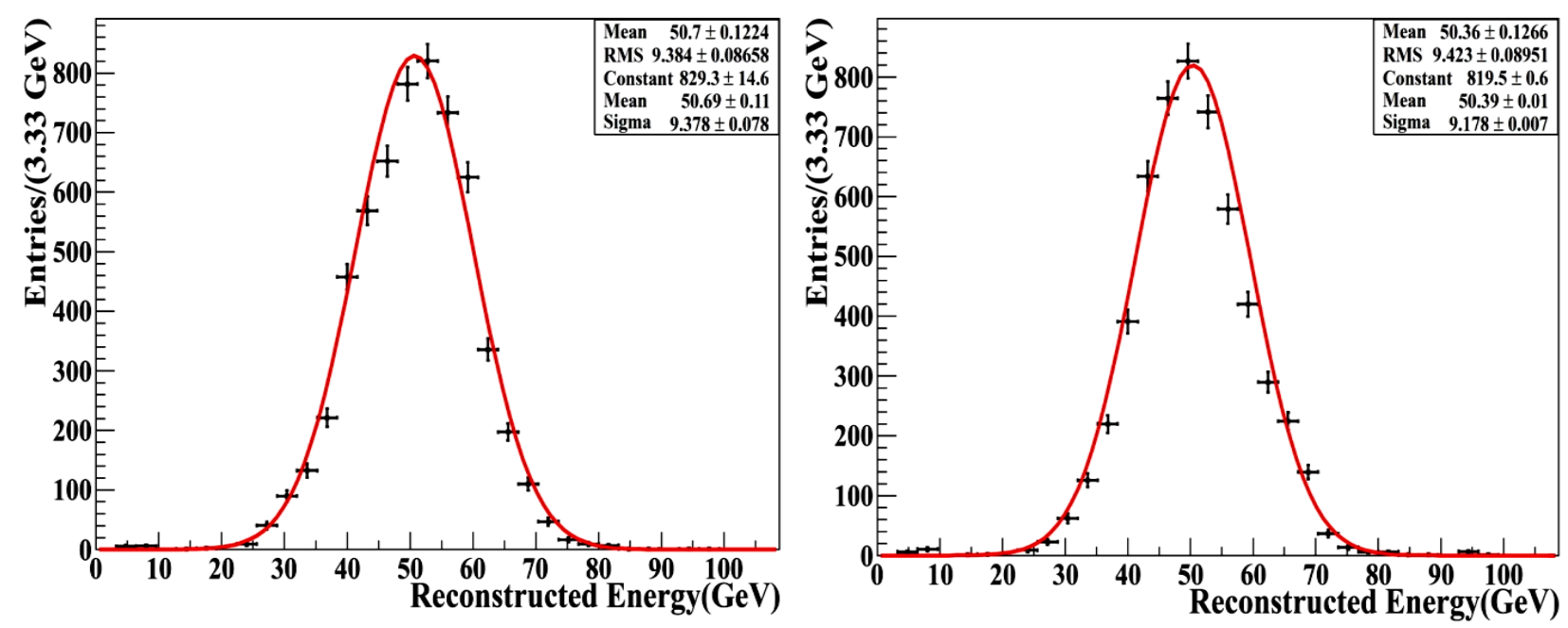

Figure 9. Distributions of reconstructed energy for $50 \mathrm{GeV}$ pions for digital readout. (a) for the first 5000 events and (b) for the events from 5000 to 10000 .
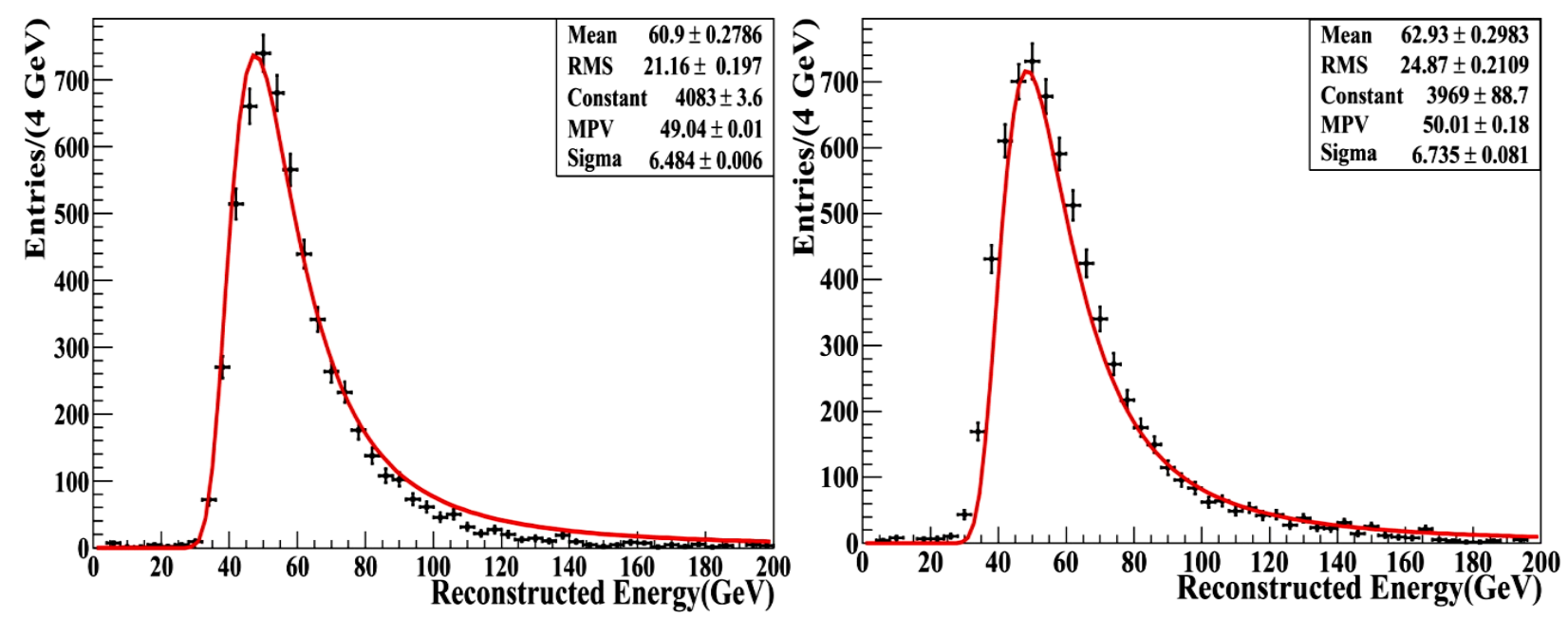

Figure 10. Distributions of reconstructed energy for $50 \mathrm{GeV}$ pions for analog readout. (a) for the first 5000 events and (b) for the events from 5000 to 10000 .

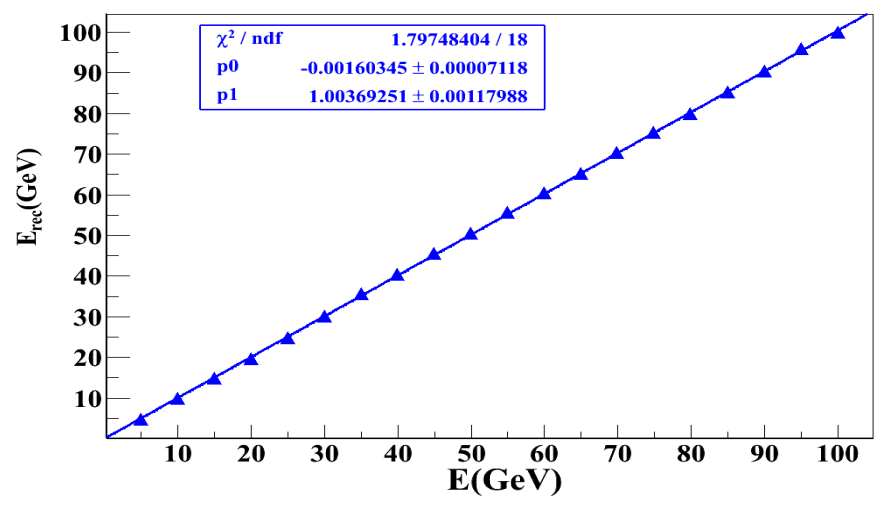

Figure 11. Linearity between the reconstructed energy and the primary pions energy for digital readout.

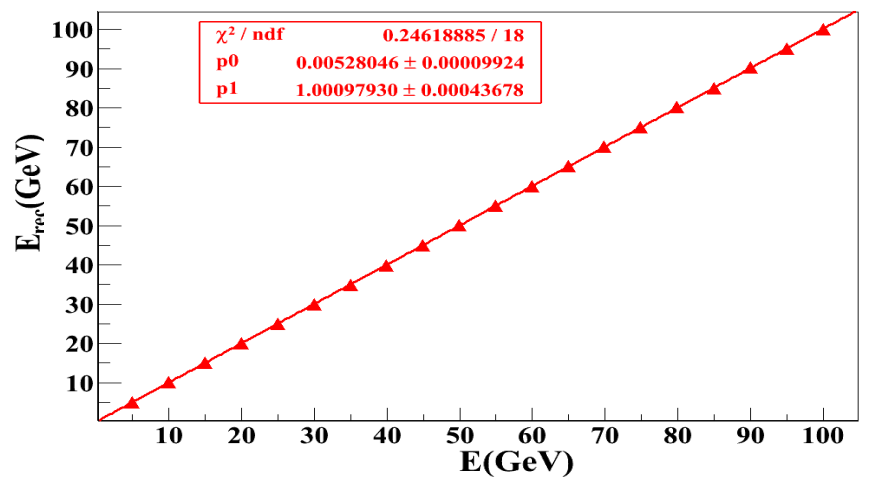

Figure 12. Linearity between the reconstructed energy and the primary pions energy for analog readout. 


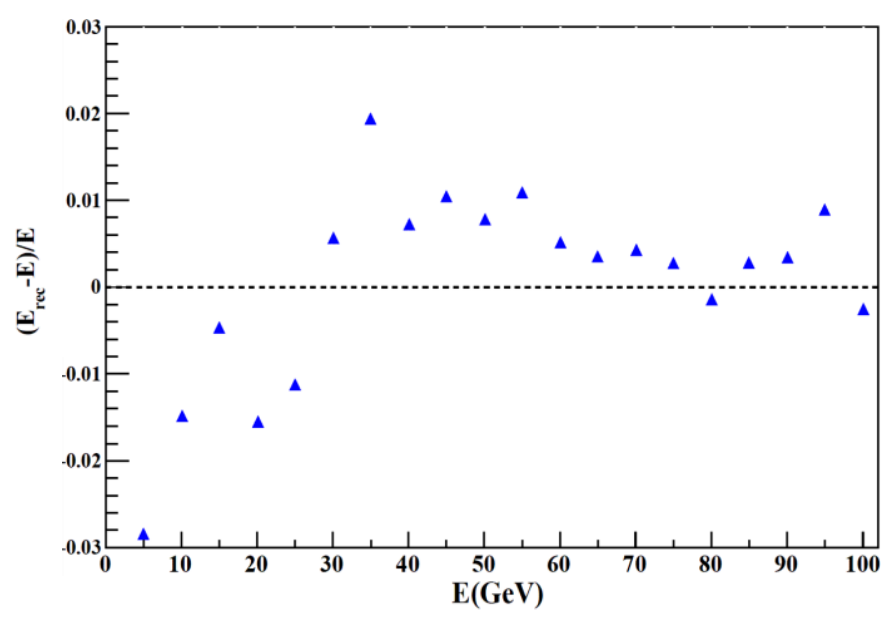

Figure 13. Full scale nonlinearity for digital readout.

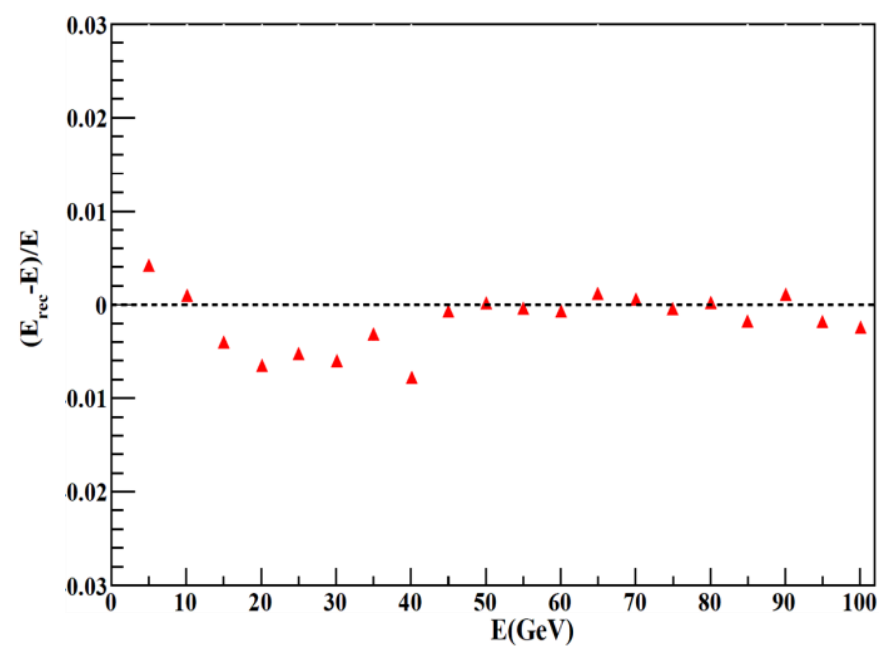

Figure 14. Full scale nonlinearity for analog readout.

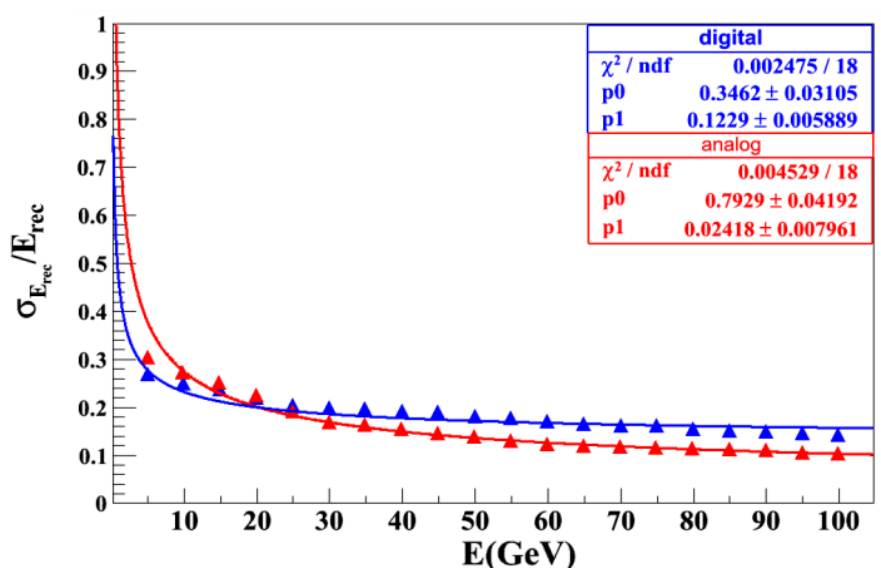

Figure 15. Comparison between the energy resolution of the digital readout and that of the analog, using reconstructed energy $\left(p_{0}=S\right.$ and $\left.p_{1}=C\right)$. $\frac{\boldsymbol{\sigma}_{\text {Erec }}}{\text { Erec }}= \begin{cases}\frac{(79.29 \pm 4.192) \%}{\sqrt{\mathrm{E}(\mathrm{GeV})}}+(2.412 \pm 0.7961) \% & \text { Analog } \\ \frac{(34.62 \pm 3.105) \%}{\sqrt{\mathrm{EGeV})}}+(12.29 \pm 0.5889) \% & \text { Digital }\end{cases}$

\section{Conclusion}

The simulation study of the HCAL characteristics which uses tungsten as an absorber and RPCs as an active medium shows that at low energies, the energy resolution is better for the digital mode than for the analog mode, and the opposite for high energies. However, the calorimeter linearity response is better for the analog mode than for the digital mode. The analog resolution is dominated only by Landau fluctuations, but in the digital mode the resolution and linearity are degraded by the saturation effect which can be suppressed by applying two or three thresholds.

These results are valid for 0.1 MIP thresholds. The effect of thresholds on the calorimeter response, the choice and the optimization thresholds for digital HCAL and the performance study for semi-digital HCAL will be the subjects of the future work. Already, we started studying on the thresholds choice and the results will be published later.

This numerical work can be reproduced for other configurations, for example we can change the type of absorber (iron, lead or steel) and the type of active medium (MICROMEGAS or GEM).

\section{REFERENCES}

Adloff C Blaha J, Blaising J, Chefdeville M, Espargilière A, Karyotakis $Y$ (2009). Monte Carlo study of the physics performance of a digital hadronic calorimeter. JINST. 4:P11009.

Agostinelli S, Allison J, Amako K (2003). Geant4 Collaboration. Nucl. Instrum. Methods Phys. Res. A 506:250-303.

Akindinov AV, Alici A, Anselmo A (2004). Study of gas mixtures and ageing of the multigap resistive plate chamber used for the Alice TOF. Nucl. Instrum. Methods Phys. Res. A 533: 93-97.

Ambrosio M, Candela A, Deo MDe, D'Incecco M, Gamba D, Giuliano A, Gustavino C, Lindozzi M, Morganti S, Redaelli N, Tonazzo A, Trapani P, Trinchero GC (2003). Aging measurements on glass RPCs. IEEE Trans. Nucl. Sci. 50(4):820-824.

Ammosov V (2002). TESLA digital hadron calorimeter (requirements, status and plans for R\&D). Nucl. Instrum. Methods Phys. Res. A 494:355-361.

Ammosov V, Gapienko V, Ivanilov A, Sefkow F, Semak A, Sviridov Yu, Usenko E, Zaets V (2004). Small pad RPCs as detector for high granularity digital hadron calorimetry. Nucl. Instrum. Methods Phys. Res. A 533:130-138.

Ammosov V, Gapienko V, Ivanilov A, Semak A, Sviridov Yu, Zaets V (2007). Study of RPCs with $1 \times 1 \mathrm{~cm}^{2}$ read-out pads operated in the saturated avalanche mode. IHEP. P. 22.

Andreev V, Balagura V, Bobchenko B, Buzhan P (2005). A highgranularity scintillator calorimeter readout with silicon photomultipliers. Nucl. Instrum. Methods Phys. Res. A 540:368-380.

Andreev V, Cvach J, Danilov M, Devitsin E (2006). A high-granularity plastic scintillator tile hadronic calorimeter with APD readout for a linear collider detector. Nucl. Instrum. Methods Phys. Res. A 564:144-154. 
Bedjidian M, Belkadhi K, Boudry V, Combaret C (2010). Performance of Glass Resistive Plate Chambers for a high-granularity semi-digital calorimeter. JINST 6:P02001.

Bilki B, Butler J, Cundiff T, Drake G (2008). Calibration of a digital hadron calorimeter with muons. JINST 3:P05001.

Bilki B, Butler J, May E, Mavromanolakis G, Norbeck E, Repond J, Underwood D, Xia L, Zhang Q (2009). Measurement of the rate capability of Resistive Plate Chambers. JINST. 4: P06003.

Calcaterra A, Sangro R de, Gamba D, Mannocchi G (2004). Test of large area glass RPCs at the DAФNE Test Beam Facility (BTF). Nucl. Instrum. Methods Phys. Res. A 533:154-158.

Camarri P, Cardarelli R, Ciaccio ADi, Santonico R (1998). Streamer suppression with SF6 in RPCs operated in avalanche mode. Nucl. Instrum. Methods Phys. Res. A. 414:317-324.

Candela A, Deo MDe, Giovanni ADi, Marco NDi, D'Incecco M, Gustavino C, Redaelli N, Tonazzo A, Trinchero GC (2004). Ageing and recovering of glass RPC. Nucl. Instrum. Methods Phys. Res. A 533:116-120.

Doroba K (2004). Precision test of electroweak interactions-what we have learned from LEP and SLC. Acta Phys. Pol. B 35:1173-1189.

Drake G, Repond J, Underwood S, Xia L (2007). Resistive Plate Chambers for hadron calorimetry: Tests with analog readout. Nucl. Instrum. Methods Phys. Res. A 578:88-97.

Fonte $P$ (2002). Applications and new developments in resistive plate chambers IEEE Trans. Nucl. Sci. 49(3):881-887.

ILC Reference Design Report (RDR), ILC Collaboration, ILC-REPORT2007-001. ILC Homepage: http://www.linearcollider.org/cms/.

Leroy C, Rancoita PG (2000). Physics of cascading shower generation and propagation in matter: Principles of high-energy, ultrahigh-energy and compensating calorimetry. Rep. Prog. Phys. 63:505-606.
Magill S (2003). Comparison of simulated analog versus digital energy measurement in a finely-segmented hadron calorimeter P. 009.

Repond J (2007). Calorimetry at the International Linear Collider. Nucl. Instrum. Methods Phys. Res. A 572:211-214.

ROOT, an object-oriented data analysis framework, version 5.30.01. CERN, 2011. Available:http://root.cern.ch.

The Geant4 web page: http://cern.ch/geant4.

Thomson MA (2009). Particle Flow Calorimetry and the PandoraPFA Algorithm. Nucl. Instrum. Methods Phys. Res. A 611:25-40.

Va'vra J (2003). Summary of session 6: Aging effects in RPC detectors. Nucl. Instrum. Methods Phys. Res. A 515:354-357.

Wigmans R (2000). Calorimetry, Energy Measurement in Pariticle Physics. New York, Oxford University Press. 